Paper published in Schizophrenia Research online:

https://doi.org/10.1016/j.schres.2020.03.017

\title{
Altered diffusion in motor white matter tracts in psychosis patients with
}

\section{catatonia}

Petra V. Viher ${ }^{1}$, Katharina Stegmayer ${ }^{1}$, Andrea Federspiel ${ }^{1}$, Stephan Bohlhalter ${ }^{2,3}$, Roland Wiest ${ }^{4}$ Sebastian Walther ${ }^{1}$

${ }^{1}$ Translational Research Center, University Hospital of Psychiatry and Psychotherapy, University of Bern, Bern, Switzerland;

${ }^{2}$ Department of Clinical Research, University Hospital, Inselspital, Bern, Switzerland;

${ }^{3}$ Neurocenter, Luzerner Kantonsspital, Lucerne, Switzerland;

${ }^{4}$ Support Center of Advanced Neuroimaging, Institute of Diagnostic and Interventional Neuroradiology, University of Bern, Bern, Switzerland

Corresponding author:

Prof. Sebastian Walther, M.D.

University Hospital of Psychiatry

Bolligenstrasse 111, 3000 Bern 60, Switzerland

sebastian.walther@upd.unibe.ch

Phone: +41319309757

Fax: +41319309404 
Abstract

Catatonia is a complex psychomotor symptom frequently observed in schizophrenia. Neural activity within the motor system is altered in catatonia. Likewise, white matter (WM) is also expected to be abnormal. The aim of this study was to test, if schizophrenia patients with catatonia show specific WM alterations. Forty-eight patients with schizophrenia and 43 healthy controls were included. Catatonia was currently present in 13 patients with schizophrenia. Tract-Based Spatial Statistics was used to test for differences in fractional anisotropy (FA) in the whole brain between the three groups. We detected a group effect (F-test) of WM within the corpus callosum (CC). In the $T$-test, patients with catatonia showed higher FA in many left lateralized WM clusters involved in motor behaviour compared to patients without catatonia, including the $\mathrm{CC}$, internal and external capsule, superior longitudinal fascicle (SLF) and corticospinal tract (CST). Similarly, patients with catatonia showed also higher FA in the left internal capsule and left CST compared to healthy controls. In contrast, the group comparison between patients without catatonia and healthy controls revealed lower FA in many right lateralized clusters, comprising the CC, internal capsule, SLF, and inferior longitudinal fascicle in patients without catatonia. Our results are in line with the notion of an altered motor system in catatonia. Thus, our study provides evidence for increased WM connectivity, especially in motor tracts in schizophrenia patients with catatonia.

Keywords: Schizophrenia; Diffusion tensor imaging; Tract-Based Spatial Statistics; Motor system; Catatonia.

\section{Introduction}

Catatonia is a complex psychomotor syndrome and was first introduced by the German psychiatrist Karl Ludwig Kahlbaum in 1874 (Kahlbaum, 1874). The catatonia syndrome comprises a range of psychomotor phenomena, including altered volition (e.g. negativism, automatic obedience), aberrant motor activity, such as increases (e.g. excitement and agitation), decreases (e.g. stupor, staring) or abnormalities (e.g. posturing, grimacing, waxy flexibility), as well as autonomic dysfunction (e.g. hyperthermia, tachycardia) (Walther et al., 2019). Notably, catatonia can be related to various motor signs and symptoms, even though no specific symptom identifies catatonia (Walther and Strik, 2016). Although catatonia has often been associated with schizophrenia, it also occurs in various psychiatric and medical conditions (Daniels, 2009; Fink, 2013). This was acknowledged in the Diagnostic and Statistical Manual of Mental Disorders, Fifth Edition (DSM-5). According to DSM-5, the catatonia 
syndrome may now be diagnosed in many psychiatric disorders, including psychotic disorders, major mood disorders, medical conditions and as not otherwise defined (Walther and Strik, 2016).

The prevalence rate of catatonia in schizophrenia is high, but depends largely on the criteria applied in diagnostic systems and is estimated between 9-18 \% (Walther et al., 2019). The presence of catatonia in schizophrenia is associated with more negative symptoms and indicates poorer course of the illness and prognosis in the chronic phase (Ungvari et al., 2005; van den Ameele et al., 2015).

The pathobiology of catatonia is still largely unknown (Walther and Strik, 2016). However, the understanding of neurobiological processes would allow a more accurate characterisation of catatonia (van den Ameele et al., 2015) and shed light on the question, whether catatonia describes a general clinical phenotype with different underlying mechanisms or a common phenotype with one pathobiology (Walther et al., 2019; Walther and Strik, 2016). Alterations in cortical and subcortical motor areas are thought to give rise to catatonia symptoms (Hirjak et al., 2019; Hirjak et al., 2015; Mittal et al., 2017; van Harten et al., 2017; Walther, 2015; Walther et al., 2019).

A few studies investigated task-related and resting state activity in catatonia and detected altered patterns of activity in motor regions related to catatonia (Foucher et al., 2018; Northoff et al., 1999; Northoff et al., 2000; Payoux et al., 2004; Scheuerecker et al., 2009; Walther et al., 2017a). However, white matter (WM) correlates of catatonia are largely unknown. In schizophrenia, this is of particular interest, as WM abnormalities may contribute to the pathophysiology of schizophrenia and altered brain connectivity is suggested as a central characteristic of the disorder (Davis et al., 2003). Thus, WM alterations have frequently been observed in schizophrenia, predominantly in the WM of the frontal and temporal areas and in fiber tracts which connect those regions (Federspiel et al., 2006; Fitzsimmons et al., 2013; Kubicki et al., 2007; Kyriakopoulos et al., 2008). More precisely, decreased fractional anisotropy (FA) has been reported in the arcuate fascicle (AF), corpus callosum, cingulum bundle, fornix, inferior longitudinal fascicle (ILF), superior longitudinal fascicle (SLF), and uncinate fascicle (UF) (Whitford et al., 2011).

One study with putative catatonia genotype suggests, that WM alterations of the corpus callosum may be associated to the catatonia syndrome (Hagemeyer et al., 2012). The aim of our study was to investigate possible WM alterations linked to current catatonia in a sample of patients with schizophrenia spectrum disorders. We hypothesized WM alterations in the group of schizophrenia patients with catatonia compared to the group without catatonia and compared to healthy controls, predominantly in motor tracts. Furthermore, we hypothesized a direct association between catatonia severity and WM abnormalities. 


\section{Methods}

\subsection{Participants}

The sample included 48 patients with schizophrenia ( 30 men, 18 women) from the inpatient and outpatient department of the University Hospital of Psychiatry in Bern, Switzerland, and 43 healthy participants ( 25 men, 18 women), matched for sex, age, and duration of education. All participants were right-handed as tested by the Edinburgh handedness inventory (Oldfield, 1971).

All patients met the inclusion criteria of a diagnosis of schizophrenia spectrum disorders (including schizophrenia, schizoaffective disorder or schizophreniform disorder) according to DSM-5. Healthy controls had specific exclusion criteria, i.e. history of any psychiatric disorder or any first-degree relatives with schizophrenia. For all participants, additional exclusion criteria comprised aberrant movement or WM due to medical or neurological conditions (e.g. multiple sclerosis, stroke), a history of head trauma with loss of consciousness, electroconvulsive treatment, any substance-related addiction (except nicotine), and distinct exclusion criteria for the magnetic resonance imaging (MRI) scanning (e.g. claustrophobia, metallic implants or pregnancy).

Catatonia symptoms were determined with the Bush Francis Catatonia Rating Scale (BFCRS) (Bush et al., 1996). This rating scale consists of 23 items (e.g. automatic abnormality, excitement, mutism, perseveration). The BFCRS Screening Instrument (BFCRSI) includes the first 14 items of the BFCRS. Current catatonia is present, if 2 or more items on the BFCRSI last longer than 24 hours (Bush et al., 1996).

Additional assessments comprised the Comprehensive Assessment of Symptoms and History (CASH) (Andreasen et al., 1992) and the Mini International Neuropsychiatric Interview (MINI) (Sheehan et al., 1998) to ascertain diagnoses according to DSM-5 and the Positive and Negative Syndrome Scale (PANSS) (Kay et al., 1987) for schizophrenia psychopathology. Current antipsychotic medication dosage was assessed as chlorpromazine equivalents (CPZ) according to Leucht and colleagues (Leucht et al., 2015). Forty-four patients (92\%) were treated with antipsychotics.

The protocol was in accordance with the Declaration of Helsinki and was approved by the "Kantonale Ethikkommission Bern" (KEK-BE 025/13). Written informed consent was obtained from all participants. The responsible psychiatrists confirmed the capacity of the patients to provide informed consent.

\subsection{MRI acquisition}

Images were acquired on a 3 T whole body MRI scanner (Siemens Magnetom Trio; Siemens Medical Solutions, Erlangen, Germany). A 12-channel headcoil was used for signal reception. Diffusion tensor 
imaging (DTI) measurements were acquired with a spin echo planar imaging (EPI) sequence (TR $8 \mathrm{~s}$, TE $92 \mathrm{~ms}$, 59 slices, FOV $=256 \times 256 \mathrm{~mm}^{2}$, matrix size $128 \times 128,2 \mathrm{~mm}$ slice thickness, gap between slices $=0 \mathrm{~mm}$, producing a $2 \mathrm{~mm}^{3}$ isotopic voxel resolution) which covers the whole brain (bandwidth $1346 \mathrm{~Hz} / \mathrm{Px}, 40 \mathrm{mT} / \mathrm{m}$ gradient, GRAPPA factor 2, 6/8 partial Fourier). The axial slices were located in the parallel plane to the AC-PC line and acquired along 42 directions using a $b$-value $=1300 \mathrm{~s} / \mathrm{mm}^{2}$. A diffusion-encoding scheme, which is rotationally invariant and balanced was used to measure the DTI data. Acquisition time lasted 6 minutes.

\subsection{DTI processing}

DTI analyses were performed with the Tract-Based Spatial Statistics (TBSS) software (Smith et al., 2006; Smith et al., 2004), which is implemented in the FMRIB (Functional Magnetic Resonance Imaging of the Brain's diffusion toolbox) Software Library (FSL) (http://www.fmrib.ox.ac.uk/fsl). First, all scans were checked visually for image quality and orientation. All images were then motion corrected with the FSL tool "eddy". Eddy current-induced distortions were equally corrected with the tool "eddy". This tool is standard in FSL and reduces distortions (eddy currents and subject movements) by using affine alignment for each diffusion weighted image to the $b_{0}$ image. A tensor model was applied on the raw data to create FA images and then a brain extraction tool was performed (Smith, 2002). The FA image of each subject was aligned and transformed to a $1 \mathrm{~mm}^{3}$ Montreal Neurological Institute (MNI) standard space (Andersson et al., 2007a; Andersson et al., 2007b), using a b-spline representation of the registration warp field (Rueckert et al., 1999). The mean of all FA images created a thinned, mean FA skeleton. The aligned FA maps of each subject were projected on the skeleton. A FA threshold of 0.2 was utilized to facilitate the inclusion of only skeletal voxels. Voxel-wise between subject statistics was performed for each point on the skeleton. Other parameters of DTI, including axial diffusivity (AD), mean diffusivity (MD) and radial diffusivity (RD) were similarly calculated. Applying the "non_fa" option in TBSS, the nonlinear warps and skeleton projection of the FA images were applied to $A D, M D$ and RD.

\subsection{Statistical analysis}

TBSS was used to analyse WM microstructure. In TBSS, general linear models (GLM), based on nonparametric permutation test theory, were applied (Smith et al., 2006). F-test and $T$-tests were performed in the GLM framework. We first looked for significant differences in WM between the three groups with an F-test. In a second step, we applied three $T$-tests between the three groups. In the two $T$-tests between patients with schizophrenia and controls we entered age as a covariate of no interest, and in the $T$-test between the patients with vs. without catatonia we entered age and current CPZ dosage as covariates of no interest into our analyses. Lastly, we selected all patients with schizophrenia who presented with catatonia according to the BFCRS and calculated a correlation 
between BFCRS and FA, controlling for age and CPZ. However, to increase the sample size for correlational analyses, we also included patients with schizophrenia who displayed a value above 0 on the BFCRS (i.e. any catatonia symptom), resulting in a sample size of 16.

Differences in WM were examined with various parameters of $D T I$, including $F A, A D, M D$ and RD. Applying a randomise tool, voxelwise differences in $F A, A D, M D$ and $R D$ between groups were tested (Winkler et al., 2014) (5’000 permutations). This tool uses a threshold-free cluster enhancement (TFCE) correction (Smith and Nichols, 2009), whereby a $p$-value < 0.05 (FWE corrected) indicates statistical significance. The locations of the significant clusters were anatomically determined by using the Johns Hopkins University (JHU)-ICBM-DTI-81 WM labels atlas and the JHU-WM tractography atlas (Mazziotta et al., 2001; Mori et al., 2005b). Only clusters with a size $>30$ voxels are presented. For illustration purposes, we used the tbss_fill option in FSL for the figures. (http://www.fmrib.ox.ac.uk/fsl/tbss/index.html).

\section{Results}

Catatonia was present in 13 patients with schizophrenia ( $\geq 2$ items on the BFCRSI for a minimum of 24 hours). Patients with catatonia and without are comparable in duration of illness, chlorpromazine equivalents (CPZ) and PANSS positive syndrome score. However, patients with catatonia had increased PANSS negative syndrome and total scores compared to patients without catatonia. The demographic and clinical information are given in Table 1.

\subsection{WM differences across groups}

Using an F-test, we found significant alterations in FA across the 3 groups in the splenium and body of the corpus callosum ( $p<0.05$, FWE corrected) (see Figure 1 and supplementary Table S1). We further detected differences in RD across all groups including the SLF and corticospinal tract (CST) (see supplementary Table S2).

\subsection{WM differences between patients with and without catatonia}

We examined a $T$-Test between patients with vs. patients without catatonia and included age and CPZ as variables of no interest. The analysis detected significant group differences in many WM clusters, comprising the corpus callosum, cingulum, internal and external capsule, CST, sagittal stratum, SLF, ILF, and inferior-fronto-occipital fascicle (IFOF). Overall, the significant clusters were more left lateralized. In all these fiber tracts, patients with catatonia revealed significantly higher FA 
values compared to patients without catatonia ( $p<0.05$, FWE corrected) (see Figure 2 and supplementary Table S3). Similar regions were detected in the identical analysis for RD. In this TTest, patients with catatonia showed lower RD values than patients without catatonia (see supplementary Table S4).

\subsection{WM differences between patients with catatonia and healthy controls}

Comparing patients with catatonia vs. healthy controls with age as a variable of no interest, we detected significant differences in WM in the left internal capsule, left posterior corona radiata and left CST ( $p<0.05$, FWE corrected). In all WM connections, patients with catatonia revealed higher FA values than healthy controls (see Figure 3 and supplementary Table S5).

\subsection{WM differences between patients without catatonia and healthy controls}

Patients without catatonia had reduced FA values than healthy controls in several right-lateralized clusters (see supplementary Table S6 and Figure S1). In addition, the same analyses for MD and RD showed significantly higher RD and in a lesser extent for MD in similar WM clusters in patients without catatonia ( $p<0.05$, FWE corrected) (see supplementary Tables S7 and S8).

\subsection{Associations of WM with catatonia severity}

The GLM performed in TBSS revealed no significant associations in a subsample of 16 patients with catatonia (values >0 on BFCRS) between the BFCRS score and WM (MD $(p=0.074) ; \operatorname{RD}(p=0.076)$, FA $(p=0.154)$, all FWE corrected). In line with the other results, we observe a positive association for FA, indicating the higher the FA, the more catatonia severity. The clusters (not FWE corrected) include similar regions and are again more left lateralized (data not shown). The associations between MD or RD with the BFCRS score were negative.

\section{Discussion}

This study investigated WM abnormalities in schizophrenia patients with catatonia. The results indicate specific left-lateralized WM alterations of motor pathways in catatonia. Compared to healthy controls, patients with catatonia revealed higher FA in the CST and internal capsule. Increased FA of the same tracts and of additional pathways, for example of the corpus callosum, SLF and external capsule were also observed in patients with catatonia in comparison to patients without catatonia. Thus, our results substantiate altered cerebral motor circuits as a putative mechanism in catatonia in the context of schizophrenia (Walther et al., 2019). 
Our results of altered white matter in motor pathways in catatonia are in line with studies reporting altered task-related activity in catatonia. For example, compared to controls catatonia patients had decreased neural activity during sequential finger movement tasks in the supplementary motor area (SMA), premotor cortex, primary sensorimotor cortex, and bilateral inferior parietal lobe (Northoff et al., 1999; Payoux et al., 2004). Likewise, during self-initiated movements catatonia patients presented a hypoactivation in the SMA, the prefrontal and parietal areas (Scheuerecker et al., 2009). In addition, patients with catatonia had alterations in orbitofrontal cortex activity and in functional connectivity between the premotor cortex and orbitofrontal cortex during negative and positive emotional stimulation compared to other psychiatric controls and healthy controls (Northoff et al., 2004).

Altered resting state activity has also been reported in catatonia. Single case studies detected altered perfusion in the frontal, temporal and parietal areas and in subcortical regions (stratum, thalamus) (Galynker et al., 1997; Tsujino et al., 2011). Similarly, one study with 10 patients with catatonia showed hypoperfusion in the right prefrontal and parietal cortex (Northoff et al., 2000). Severe catatonia symptoms were correlated with resting state functional thalamocortical connectivity in patients with schizophrenia (Walther et al., 2017b). Recently, hyperperfusion of the SMA and primary motor cortex (M1) was reported in patients with catatonia at rest (Walther et al., 2017a). This finding was corroborated by a second study reporting increased CBF in SMA and M1 in another sample of schizophrenia patients with catatonia (Foucher et al., 2018).

The motor system may be characterized by states of excitation, which can be captured by dynamic models of brain function (Walther, 2015). Hence, structural correlates of the catatonia syndrome may be more difficult to identify. A recent study detected structural alterations in patients with catatonia compared to patients without catatonia, i.e. reduced surface area and altered gyrification in the parietal and frontal regions (Hirjak et al., 2019). In addition, cortical thinning of frontal and insular areas were related to catatonia symptoms (Hirjak et al., 2019).

A great strength of the present study is, that it succeeded in mapping the catatonia syndrome to brain structure, particularly the major white matter pathways of the motor system. Interestingly, the WM regions of all analyses concerning catatonia were more left lateralized. In contrast, the only analysis not relating to catatonia (contrast between patients without catatonia and healthy controls) revealed significant clusters more right lateralized. In addition, this contrast did not include some specific fiber tracts in comparison to the contrast between the patient sample, for example the external capsule, sagittal stratum, cingulum, left SLF and left posterior limb of the internal capsule. Indeed, the catatonia syndrome seems to be particularly related to the left CST and left internal capsule, as revealed by the contrast between patients with catatonia vs. healthy controls. Thus, 
alterations in motor pathways were more left lateralized in our sample of right-handed catatonia patients.

Generally, patients with schizophrenia show reduced FA values compared to healthy controls (Vitolo et al., 2017). In contrast, in this study schizophrenia patients with catatonia presented significantly higher FA values in several WM regions compared to the other groups. While the patients with catatonia had the highest overall FA values, the patients without catatonia had the lowest overall FA values and the healthy controls are situated in the middle. Thus, the contrast between patients with vs. without catatonia showed the greatest differences in FA.

We detected alterations of specific motor pathways, including the CST, SLF, internal capsule and corpus callosum in patients with catatonia. The CST contains descending fibers from the precentral motor area to the pons and medulla (Mori et al., 2005a) and is a central pathway for voluntary motor control (Martin, 2005). The internal capsule includes the thalamocortical and long corticofugal tracts and can be split into the anterior limb, posterior limb and retrolenticular part (Mori et al., 2005a). It is a very important projection system for perceptual and motor functions (Catani and Thiebaut de Schotten, 2008). The SLF is situated at the side of putamen, runs along the superior edge of the insula and forms the arcuate fascicle, which runs into the frontal, parietal, temporal and occipital lobes (Mori et al., 2005a). The SLF regulates higher order motor behaviour (Makris et al., 2005). Interhemispheric motor fibers, which project in premotor, SMA and primary motor cortex are found in the body of the CC (Hofer and Frahm, 2006). The corpus callosum is crucially involved in the processing of sensory, cognitive and motor information (Perez and Cohen, 2009). In sum, catatonia patients had specifically increased FA values in relevant motor fiber pathways. However, in contrast to the categorical differences, we did not find a correlation of white matter and dimensional catatonia severity in a subsample of patients with catatonia. Since the correlations for radial diffusivity and mean diffusivity showed a trend $(p<0.1)$ in a smaller group of patients, we conclude that in larger sample sizes these results would survive FWE correction.

Our results are paralleled by one case study, reporting parietal and frontal subcortical WM alterations in a patient with catatonia (Tibrewal et al., 2017). Likewise, our findings are corroborated by another study investigating the association between a myelin gene CNP $\left(2^{\prime}, 3^{\prime}\right.$-cyclic nucleotide $3^{\prime}$ phosphodiesterase) polymorphism and catatonia (Hagemeyer et al., 2012). Reduced CNP expression was linked to distinct behavioural abnormalities as seen in catatonia and increased axial diffusivity in the context of axonal loss in the frontal corpus callosum in mice and men (Hagemeyer et al., 2012). In our sample, we report a link of higher FA in the corpus callosum with catatonia. Axial diffusivity and fractional anisotropy both describe more diffusion along the axon. However, in our sample, we have 
no genetic data to test whether our clinically established catatonia diagnoses correspond to the AAGenotype of the CNP polymorphism.

We speculate, that the catatonia syndrome in schizophrenia is related to altered WM in tracts of the motor system. Catatonia states may present with severely increased or decreased motor activity or rapid changes between both. Still, most patients display reduced motor activity (Wilson et al., 2015). The question remains, whether alterations of the motor system are cause or result of reduced quantity of movement (Walther et al., 2009). Motor training may modulate brain WM structure (Hanggi et al., 2010). For example, professional ballet dancers showed reduced WM in motor tracts, including the CST, internal capsule and corpus callosum (Hanggi et al., 2010). Similarly, skilled golfers compared to less skilled golfers revealed reduced WM volume or FA of the CST, corpus callosum and internal capsule (Jancke et al., 2009). Likewise, externally caused immobility of a limb after fracture may change brain structure within the motor system (Langer et al., 2012). Given that the catatonia patients in our sample had substantial durations of illness, we may conclude that reduced motor behaviour for longer periods of time could have shaped WM motor tracts or these alterations may follow massively increased motor behaviour. The alternative explanation would be that functional changes of the motor system may subsequently stimulate reorganization of WM pathways in schizophrenia patients with catatonia (Walther et al., 2019). Our previous study noted increased neural activity in the supplementary motor area (SMA) in subjects with massive behavioral inhibition (Walther et al., 2017a). The present findings link catatonia to increased white matter properties in the major motor fiber pathways, suggesting plastic changes in these pathways. Interestingly, the regions of neural hyperactivity in the premotor cortex are located adjunct to the altered pathways and are expected to feed these pathways. Thus, we may have observed the result of massive inhibitory activity that increases brain perfusion in the SMA and leads to plastic changes of the corresponding fiber pathways, particularly the pathways linking the SMA with the subthalamic nucleus, the so-called hyperdirect pathway (Walther et al., 2019). However, whether the changes are cause or consequence of catatonia can only be tested in longitudinal study designs. From a clinical perspective, these considerations of the current findings in white matter would call for adjunct exercise therapies in catatonia patients (Takahashi et al., 2019).

Only a few studies related motor symptoms to WM alterations in schizophrenia. For example, activity level in schizophrenia has been associated with WM alterations underneath the SMA and precentral gyrus and with fibers connecting the primary motor area with the subcortical regions and the preSMA and motor regions (Bracht et al., 2013; Walther et al., 2011). In addition, tardive dyskinesia (TD) has been associated with FA decreases in the WM of subcortical regions, postcentral gyrus, frontal cingulate gyrus, external capsule and inferior frontal gyrus WM (Bai et al., 2009). Neurological soft 
signs (NSS) have been linked to WM alterations of the corpus callosum, inferior frontal gyrus, and cerebellum in schizophrenia (Mouchet-Mages et al., 2011; Thomann et al., 2009). Similarly, WM integrity of the cerebellar-thalamic tract at baseline predicted NSS after one year in subjects at high clinical risk for psychosis (Mittal et al., 2014). Moreover, patients with schizophrenia with a motor dexterity task deficit revealed aberrant FA in WM fiber tracts, including the corpus callosum, IFOF and internal capsule (Hidese et al., 2018; Perez-Iglesias et al., 2010). Finally, the severity of the DSM5 motor dimension has been associated with WM integrity of motor tracts (e.g. superior longitudinal fascicle, internal capsule) in schizophrenia (Viher et al., 2016).

The present study has some limitations. First, the study included only patients with schizophrenia in order to investigate the catatonia syndrome. This does not take into account that catatonia is also present in other disorders, such as major mood disorders or in medical conditions. Second, we included a small sample of 13 patients with catatonia in our study. However, it is one of the larger samples of catatonia patients ever analysed with brain imaging methods, and it is one of the first studies using a whole brain approach to investigate white matter alterations in catatonia. Third, some variables may affect the integrity of WM. While a number of studies could not confirm an association of antipsychotic treatment and WM alterations (Kanaan et al., 2009; Kraguljac et al., 2019; Peters et al., 2010), including medication- naïve chronic patients (Liu et al., 2013), there are some hints for a medication effect. For example, antipsychotic exposure has been shown to affect free radicals from activated microglia, the release of inflammatory cytokines and the number of astrocytes in monkeys (Konopaske et al., 2008; Monji et al., 2009). In addition, age is associated with WM, especially of the frontal and parietal structure (Davis et al., 2009; Geerligs et al., 2015; GunningDixon et al., 2009; Salat et al., 2005). Therefore, we entered age and the medication dose as covariates into the analyses in order to control for age and medication related effects on WM. Fourth, in the present study we selected a hypothesis-free whole brain approach, which allows the detection of WM changes across the brain. However, this method compares only voxels that are thought to correspond to specific tracts and does not reveal information about the tract itself. Future studies could use a hypothesis driven approach, deterministic and probabilistic tractography. Lastly, the relationship between WM integrity and catatonia symptomatology may not be straightforward. WM alterations are considered as rather stable over time, while the presence of catatonia symptoms may vary within 24 hours (Walther and Strik, 2016). Hence, a cross-sectional study design does not sufficiently consider the uniqueness of disorder courses and does further not allow conclusions about the cause-effect relationship. Longitudinal studies could unravel the association between brain structure and catatonia symptoms, as they have more predictive value.

In summary, the present study suggests substantial structural brain alterations in major motor pathways in patients with catatonia, a severe psychomotor syndrome. The current study helps to 
unravel the pathobiology of catatonia by focusing on WM changes. Future investigations should also include catatonia in non-schizophrenia cases to establish whether there is common or distinct pathobiology among catatonia syndromes.

\section{Acknowledgement}

This work was partly supported by a grant of the Swiss National Science Foundation (\#152619) to S.W., A.F. and S.B.

\section{References}

Andersson, J.L.R., Jenkinson, M., Smith, S., 2007a. Non-linear optimisation FMRIB Technical Report TR07JA1.

Andersson, J.L.R., Jenkinson, M., Smith, S., 2007b. Non-linear registration aka Spatial normalisation.

Andreasen, N.C., Flaum, M., Arndt, S., 1992. The Comprehensive Assessment of Symptoms and History (CASH). An instrument for assessing diagnosis and psychopathology. Arch Gen Psychiatry 49(8), 615-623.

Bai, Y.M., Chou, K.H., Lin, C.P., Chen, I.Y., Li, C.T., Yang, K.C., Chou, Y.H., Su, T.P., 2009. White matter abnormalities in schizophrenia patients with tardive dyskinesia: a diffusion tensor image study. Schizophr Res 109(1-3), 167-181.

Bracht, T., Schnell, S., Federspiel, A., Razavi, N., Horn, H., Strik, W., Wiest, R., Dierks, T., Muller, T.J., Walther, S., 2013. Altered cortico-basal ganglia motor pathways reflect reduced volitional motor activity in schizophrenia. Schizophr Res 143(2-3), 269-276.

Bush, G., Fink, M., Petrides, G., Dowling, F., Francis, A., 1996. Catatonia. I. Rating scale and standardized examination. Acta Psychiatr Scand 93(2), 129-136.

Catani, M., Thiebaut de Schotten, M., 2008. A diffusion tensor imaging tractography atlas for virtual in vivo dissections. Cortex 44(8), 1105-1132.

Daniels, J., 2009. Catatonia: clinical aspects and neurobiological correlates. J Neuropsychiatry Clin Neurosci 21(4), 371-380.

Davis, K.L., Stewart, D.G., Friedman, J.I., Buchsbaum, M., Harvey, P.D., Hof, P.R., Buxbaum, J., Haroutunian, V., 2003. White matter changes in schizophrenia: evidence for myelin-related dysfunction. Arch Gen Psychiatry 60(5), 443-456.

Davis, S.W., Dennis, N.A., Buchler, N.G., White, L.E., Madden, D.J., Cabeza, R., 2009. Assessing the effects of age on long white matter tracts using diffusion tensor tractography. Neuroimage 46(2), 530-541.

Federspiel, A., Begre, S., Kiefer, C., Schroth, G., Strik, W.K., Dierks, T., 2006. Alterations of white matter connectivity in first episode schizophrenia. Neurobiol Dis 22(3), 702-709.

Fink, M., 2013. Rediscovering catatonia: the biography of a treatable syndrome. Acta Psychiatr Scand Suppl(441), 1-47.

Fitzsimmons, J., Kubicki, M., Shenton, M.E., 2013. Review of functional and anatomical brain connectivity findings in schizophrenia. Curr Opin Psychiatry 26(2), 172-187.

Foucher, J.R., Zhang, Y.F., Roser, M., Lamy, J., De Sousa, P.L., Weibel, S., Vidailhet, P., Mainberger, O., Berna, F., 2018. A double dissociation between two psychotic phenotypes: Periodic catatonia and cataphasia. Prog Neuropsychopharmacol Biol Psychiatry 86, 363-369. 
Galynker, II, Weiss, J., Ongseng, F., Finestone, H., 1997. ECT treatment and cerebral perfusion in Catatonia. J Nucl Med 38(2), 251-254.

Geerligs, L., Renken, R.J., Saliasi, E., Maurits, N.M., Lorist, M.M., 2015. A Brain-Wide Study of AgeRelated Changes in Functional Connectivity. Cereb Cortex 25(7), 1987-1999.

Gunning-Dixon, F.M., Brickman, A.M., Cheng, J.C., Alexopoulos, G.S., 2009. Aging of cerebral white matter: a review of MRI findings. Int J Geriatr Psychiatry 24(2), 109-117.

Hagemeyer, N., Goebbels, S., Papiol, S., Kastner, A., Hofer, S., Begemann, M., Gerwig, U.C., Boretius, S., Wieser, G.L., Ronnenberg, A., Gurvich, A., Heckers, S.H., Frahm, J., Nave, K.A., Ehrenreich, H., 2012. A myelin gene causative of a catatonia-depression syndrome upon aging. EMBO Mol Med 4(6), 528-539.

Hanggi, J., Koeneke, S., Bezzola, L., Jancke, L., 2010. Structural neuroplasticity in the sensorimotor network of professional female ballet dancers. Hum Brain Mapp 31(8), 1196-1206.

Hidese, S., Ota, M., Sasayama, D., Matsuo, J., Ishida, I., Hiraishi, M., Teraishi, T., Hattori, K., Kunugi, H., 2018. Manual dexterity and brain structure in patients with schizophrenia: A whole-brain magnetic resonance imaging study. Psychiatry Res Neuroimaging 276, 9-14.

Hirjak, D., Kubera, K.M., Northoff, G., Fritze, S., Bertolino, A.L., Topor, C.E., Schmitgen, M.M., Wolf, R.C., 2019. Cortical Contributions to Distinct Symptom Dimensions of Catatonia. Schizophr Bull.

Hirjak, D., Thomann, P.A., Kubera, K.M., Wolf, N.D., Sambataro, F., Wolf, R.C., 2015. Motor dysfunction within the schizophrenia-spectrum: A dimensional step towards an underappreciated domain. Schizophr Res 169(1-3), 217-233.

Hofer, S., Frahm, J., 2006. Topography of the human corpus callosum revisited--comprehensive fiber tractography using diffusion tensor magnetic resonance imaging. Neuroimage 32(3), 989994.

Jancke, L., Koeneke, S., Hoppe, A., Rominger, C., Hanggi, J., 2009. The architecture of the golfer's brain. PLoS One 4(3), e4785.

Kahlbaum, K., 1874. Die Katatonie oder das Spannungsirresein. Eine klinische Form psychischer Krankheit. A. Hirschwald, Berlin, Germany.

Kanaan, R., Barker, G., Brammer, M., Giampietro, V., Shergill, S., Woolley, J., Picchioni, M., Toulopoulou, T., McGuire, P., 2009. White matter microstructure in schizophrenia: effects of disorder, duration and medication. Br J Psychiatry 194(3), 236-242.

Kay, S.R., Fiszbein, A., Opler, L.A., 1987. The positive and negative syndrome scale (PANSS) for schizophrenia. Schizophr Bull 13(2), 261-276.

Konopaske, G.T., Dorph-Petersen, K.A., Sweet, R.A., Pierri, J.N., Zhang, W., Sampson, A.R., Lewis, D.A., 2008. Effect of chronic antipsychotic exposure on astrocyte and oligodendrocyte numbers in macaque monkeys. Biol Psychiatry 63(8), 759-765.

Kraguljac, N.V., Anthony, T., Monroe, W.S., Skidmore, F.M., Morgan, C.J., White, D.M., Patel, N., Lahti, A.C., 2019. A longitudinal neurite and free water imaging study in patients with a schizophrenia spectrum disorder. Neuropsychopharmacology 44(11), 1932-1939.

Kubicki, M., McCarley, R., Westin, C.F., Park, H.J., Maier, S., Kikinis, R., Jolesz, F.A., Shenton, M.E., 2007. A review of diffusion tensor imaging studies in schizophrenia. J Psychiatr Res 41(1-2), 15-30.

Kyriakopoulos, M., Bargiotas, T., Barker, G.J., Frangou, S., 2008. Diffusion tensor imaging in schizophrenia. Eur Psychiatry 23(4), 255-273.

Langer, N., Hanggi, J., Muller, N.A., Simmen, H.P., Jancke, L., 2012. Effects of limb immobilization on brain plasticity. Neurology 78(3), 182-188.

Leucht, S., Samara, M., Heres, S., Patel, M.X., Furukawa, T., Cipriani, A., Geddes, J., Davis, J.M., 2015. Dose Equivalents for Second-Generation Antipsychotic Drugs: The Classical Mean Dose Method. Schizophr Bull 41(6), 1397-1402.

Liu, X., Lai, Y., Wang, X., Hao, C., Chen, L., Zhou, Z., Yu, X., Hong, N., 2013. Reduced white matter integrity and cognitive deficit in never-medicated chronic schizophrenia: a diffusion tensor study using TBSS. Behav Brain Res 252, 157-163. 
Makris, N., Kennedy, D.N., McInerney, S., Sorensen, A.G., Wang, R., Caviness, V.S., Jr., Pandya, D.N., 2005. Segmentation of subcomponents within the superior longitudinal fascicle in humans: a quantitative, in vivo, DT-MRI study. Cereb Cortex 15(6), 854-869.

Martin, J.H., 2005. The corticospinal system: from development to motor control. Neuroscientist 11(2), 161-173.

Mazziotta, J., Toga, A., Evans, A., Fox, P., Lancaster, J., Zilles, K., Woods, R., Paus, T., Simpson, G., Pike, B., Holmes, C., Collins, L., Thompson, P., MacDonald, D., lacoboni, M., Schormann, T., Amunts, K., Palomero-Gallagher, N., Geyer, S., Parsons, L., Narr, K., Kabani, N., Le Goualher, G., Boomsma, D., Cannon, T., Kawashima, R., Mazoyer, B., 2001. A probabilistic atlas and reference system for the human brain: International Consortium for Brain Mapping (ICBM). Philos Trans R Soc Lond B Biol Sci 356(1412), 1293-1322.

Mittal, V.A., Bernard, J.A., Northoff, G., 2017. What Can Different Motor Circuits Tell Us About Psychosis? An RDoC Perspective. Schizophr Bull 43(5), 949-955.

Mittal, V.A., Dean, D.J., Bernard, J.A., Orr, J.M., Pelletier-Baldelli, A., Carol, E.E., Gupta, T., Turner, J., Leopold, D.R., Robustelli, B.L., Millman, Z.B., 2014. Neurological soft signs predict abnormal cerebellar-thalamic tract development and negative symptoms in adolescents at high risk for psychosis: a longitudinal perspective. Schizophr Bull 40(6), 1204-1215.

Monji, A., Kato, T., Kanba, S., 2009. Cytokines and schizophrenia: Microglia hypothesis of schizophrenia. Psychiatry Clin Neurosci 63(3), 257-265.

Mori, S., Wakana, S., Nagae-Poetscher, L.M., van Zijl, P.C.M., 2005a. MRI Atlas of Human White Matter. Elsevier, Amsterdam.

Mori, S., Wakana, S., Zijl, P.C.M., 2005b. MRI atlas of human white matter. Amsterdam, The Netherlands: Elsevier.

Mouchet-Mages, S., Rodrigo, S., Cachia, A., Mouaffak, F., Olie, J.P., Meder, J.F., Oppenheim, C., Krebs, M.O., 2011. Correlations of cerebello-thalamo-prefrontal structure and neurological soft signs in patients with first-episode psychosis. Acta Psychiatr Scand 123(6), 451-458.

Northoff, G., Braus, D.F., Sartorius, A., Khoram-Sefat, D., Russ, M., Eckert, J., Herrig, M., Leschinger, A., Bogerts, B., Henn, F.A., 1999. Reduced activation and altered laterality in two neurolepticnaive catatonic patients during a motor task in functional MRI. Psychol Med 29(4), 997-1002.

Northoff, G., Kotter, R., Baumgart, F., Danos, P., Boeker, H., Kaulisch, T., Schlagenhauf, F., Walter, H., Heinzel, A., Witzel, T., Bogerts, B., 2004. Orbitofrontal cortical dysfunction in akinetic catatonia: a functional magnetic resonance imaging study during negative emotional stimulation. Schizophr Bull 30(2), 405-427.

Northoff, G., Steinke, R., Nagel, D.C., Grosser, O., Danos, P., Genz, A., Krause, R., Boker, H., Otto, H.J., Bogerts, B., 2000. Right lower prefronto-parietal cortical dysfunction in akinetic catatonia: a combined study of neuropsychology and regional cerebral blood flow. Psychol Med 30(3), 583-596.

Oldfield, R.C., 1971. The assessment and analysis of handedness: the Edinburgh inventory. Neuropsychologia 9(1), 97-113.

Payoux, P., Boulanouar, K., Sarramon, C., Fabre, N., Descombes, S., Galitsky, M., Thalamas, C., BrefelCourbon, C., Sabatini, U., Manelfe, C., Chollet, F., Schmitt, L., Rascol, O., 2004. Cortical motor activation in akinetic schizophrenic patients: a pilot functional MRI study. Mov Disord 19(1), 83-90.

Perez, M.A., Cohen, L.G., 2009. Interhemispheric inhibition between primary motor cortices: what have we learned? J Physiol 587(Pt 4), 725-726.

Perez-Iglesias, R., Tordesillas-Gutierrez, D., McGuire, P.K., Barker, G.J., Roiz-Santianez, R., Mata, I., de Lucas, E.M., Rodriguez-Sanchez, J.M., Ayesa-Arriola, R., Vazquez-Barquero, J.L., CrespoFacorro, B., 2010. White matter integrity and cognitive impairment in first-episode psychosis. Am J Psychiatry 167(4), 451-458.

Peters, B.D., Blaas, J., de Haan, L., 2010. Diffusion tensor imaging in the early phase of schizophrenia: what have we learned? J Psychiatr Res 44(15), 993-1004. 
Rueckert, D., Sonoda, L.I., Hayes, C., Hill, D.L., Leach, M.O., Hawkes, D.J., 1999. Nonrigid registration using free-form deformations: application to breast MR images. IEEE Trans Med Imaging 18(8), 712-721.

Salat, D.H., Tuch, D.S., Hevelone, N.D., Fischl, B., Corkin, S., Rosas, H.D., Dale, A.M., 2005. Age-related changes in prefrontal white matter measured by diffusion tensor imaging. Ann $\mathrm{N} Y$ Acad Sci 1064, 37-49.

Scheuerecker, J., Ufer, S., Kapernick, M., Wiesmann, M., Bruckmann, H., Kraft, E., Seifert, D., Koutsouleris, N., Moller, H.J., Meisenzahl, E.M., 2009. Cerebral network deficits in post-acute catatonic schizophrenic patients measured by fMRI. J Psychiatr Res 43(6), 607-614.

Sheehan, D.V., Lecrubier, Y., Sheehan, K.H., Amorim, P., Janavs, J., Weiller, E., Hergueta, T., Baker, R., Dunbar, G.C., 1998. The Mini-International Neuropsychiatric Interview (M.I.N.I.): the development and validation of a structured diagnostic psychiatric interview for DSM-IV and ICD-10. J Clin Psychiatry 59 Suppl 20, 22-33;quiz 34-57.

Smith, S.M., 2002. Fast robust automated brain extraction. Hum Brain Mapp 17(3), 143-155.

Smith, S.M., Jenkinson, M., Johansen-Berg, H., Rueckert, D., Nichols, T.E., Mackay, C.E., Watkins, K.E., Ciccarelli, O., Cader, M.Z., Matthews, P.M., Behrens, T.E., 2006. Tract-based spatial statistics: voxelwise analysis of multi-subject diffusion data. Neuroimage 31(4), 1487-1505.

Smith, S.M., Jenkinson, M., Woolrich, M.W., Beckmann, C.F., Behrens, T.E., Johansen-Berg, H., Bannister, P.R., De Luca, M., Drobnjak, I., Flitney, D.E., Niazy, R.K., Saunders, J., Vickers, J., Zhang, Y., De Stefano, N., Brady, J.M., Matthews, P.M., 2004. Advances in functional and structural MR image analysis and implementation as FSL. Neuroimage 23 Suppl 1, S208-219.

Smith, S.M., Nichols, T.E., 2009. Threshold-free cluster enhancement: addressing problems of smoothing, threshold dependence and localisation in cluster inference. Neuroimage 44(1), 83-98.

Takahashi, S., Keeser, D., Rauchmann, B.S., Schneider-Axmann, T., Keller-Varady, K., Maurus, I., Dechent, P., Wobrock, T., Hasan, A., Schmitt, A., Ertl-Wagner, B., Malchow, B., Falkai, P., 2019. Effect of aerobic exercise combined with cognitive remediation on cortical thickness and prediction of social adaptation in patients with schizophrenia. Schizophr Res.

Thomann, P.A., Wustenberg, T., Santos, V.D., Bachmann, S., Essig, M., Schroder, J., 2009. Neurological soft signs and brain morphology in first-episode schizophrenia. Psychol Med 39(3), 371-379.

Tibrewal, P., Bastiampillai, T., Dhillon, R., Okungu, A., Asokan, M., 2017. Schizoaffective disorder, catatonia and white matter changes - Revisiting the microglial hypothesis. Aust N Z J Psychiatry 51(10), 1056-1057.

Tsujino, N., Nemoto, T., Yamaguchi, T., Katagiri, N., Tohgi, N., Ikeda, R., Shiraga, N., Mizumura, S., Mizuno, M., 2011. Cerebral blood flow changes in very-late-onset schizophrenia-like psychosis with catatonia before and after successful treatment. Psychiatry Clin Neurosci 65(6), 600-603.

Ungvari, G.S., Leung, S.K., Ng, F.S., Cheung, H.K., Leung, T., 2005. Schizophrenia with prominent catatonic features ('catatonic schizophrenia'): I. Demographic and clinical correlates in the chronic phase. Prog Neuropsychopharmacol Biol Psychiatry 29(1), 27-38.

van den Ameele, S., Sabbe, B., Morrens, M., 2015. [Characteristics of catatonia in schizophrenia and mood disorders]. Tijdschr Psychiatr 57(2), 94-98.

van Harten, P.N., Walther, S., Kent, J.S., Sponheim, S.R., Mittal, V.A., 2017. The clinical and prognostic value of motor abnormalities in psychosis, and the importance of instrumental assessment. Neurosci Biobehav Rev 80, 476-487.

Viher, P.V., Stegmayer, K., Giezendanner, S., Federspiel, A., Bohlhalter, S., Vanbellingen, T., Wiest, R., Strik, W., Walther, S., 2016. Cerebral white matter structure is associated with DSM-5 schizophrenia symptom dimensions. Neuroimage Clin 12, 93-99.

Vitolo, E., Tatu, M.K., Pignolo, C., Cauda, F., Costa, T., Ando, A., Zennaro, A., 2017. White matter and schizophrenia: A meta-analysis of voxel-based morphometry and diffusion tensor imaging studies. Psychiatry Res Neuroimaging 270, 8-21. 
Walther, S., 2015. Psychomotor symptoms of schizophrenia map on the cerebral motor circuit. Psychiatry Res 233(3), 293-298.

Walther, S., Federspiel, A., Horn, H., Razavi, N., Wiest, R., Dierks, T., Strik, W., Muller, T.J., 2011. Alterations of white matter integrity related to motor activity in schizophrenia. Neurobiol Dis 42(3), 276-283.

Walther, S., Horn, H., Razavi, N., Koschorke, P., Muller, T.J., Strik, W., 2009. Quantitative motor activity differentiates schizophrenia subtypes. Neuropsychobiology 60(2), 80-86.

Walther, S., Schappi, L., Federspiel, A., Bohlhalter, S., Wiest, R., Strik, W., Stegmayer, K., 2017a. Resting-State Hyperperfusion of the Supplementary Motor Area in Catatonia. Schizophr Bull 43(5), 972-981.

Walther, S., Stegmayer, K., Federspiel, A., Bohlhalter, S., Wiest, R., Viher, P.V., 2017b. Aberrant Hyperconnectivity in the Motor System at Rest Is Linked to Motor Abnormalities in Schizophrenia Spectrum Disorders. Schizophr Bull 43(5), 982-992.

Walther, S., Stegmayer, K., Wilson, J.E., Heckers, S., 2019. Structure and neural mechanisms of catatonia. Lancet Psychiatry 6(7), 610-619.

Walther, S., Strik, W., 2016. Catatonia. CNS Spectr 21(4), 341-348.

Whitford, T.J., Kubicki, M., Shenton, M.E., 2011. Diffusion tensor imaging, structural connectivity, and schizophrenia. Schizophr Res Treatment 2011, 709523.

Wilson, J.E., Niu, K., Nicolson, S.E., Levine, S.Z., Heckers, S., 2015. The diagnostic criteria and structure of catatonia. Schizophr Res 164(1-3), 256-262.

Winkler, A.M., Ridgway, G.R., Webster, M.A., Smith, S.M., Nichols, T.E., 2014. Permutation inference for the general linear model. Neuroimage 92, 381-397. 


\section{Table}

Table 1. Demographic and Clinical Data

\begin{tabular}{|c|c|c|c|c|c|c|}
\hline Variables & $\begin{array}{l}\text { Catatonia } \\
\text { Patients (13) }\end{array}$ & $\begin{array}{l}\text { Patients } \\
\text { without } \\
\text { Catatonia (35) }\end{array}$ & $\begin{array}{l}\text { Healthy } \\
\text { Controls (43) }\end{array}$ & Statistics & & \\
\hline & & & & Tests & $d f$ & $P$ \\
\hline $\operatorname{Sex}(m / f)$ & & & & $X^{2}=0.52$ & 2 & 0.772 \\
\hline Age (y) & $40.7(13.6)$ & $37.4(11.4)$ & $38.6(13.7)$ & $F=0.31$ & 2 & 0.731 \\
\hline Education (y) & $12.0(3.0)$ & $13.7(3.1)$ & $14.2(2.7)$ & $F=2.73$ & 2 & 0.071 \\
\hline $\mathrm{DOI}(\mathrm{y})$ & $17.3(13.1)$ & $11.3(12.2)$ & & $T=1.5$ & 46 & 0.142 \\
\hline $\mathrm{CPZ}$ (mg) & $612.7(272.6)$ & $514.8(419.8)$ & & $T=0.78$ & 46 & 0.440 \\
\hline $\begin{array}{l}\text { PANSS } \\
\text { positive }\end{array}$ & $17.9(7.4)$ & $18.0(6.2)$ & & $T=-0.04$ & 46 & 0.971 \\
\hline $\begin{array}{l}\text { PANSS } \\
\text { negative }\end{array}$ & $21.5(5.8)$ & $17.3(4.5)$ & & $T=2.7$ & 46 & 0.011 \\
\hline PANSS total & $83.7(14.9)$ & 69.4 (16.7) & & $T=2.1$ & 46 & 0.046 \\
\hline BFCRS total & $7.2(3.9)$ & $0.1(0.4)$ & & $T=6.6$ & 12 & 0.001 \\
\hline
\end{tabular}

Note: BFCRS, Bush Francis Catatonia Rating Scale; CPZ, chlorpromazine equivalence; DOI, duration of illness; PANSS, Positive and Negative Syndrome Scale. Significant values are highlighted in bold.

\section{Figures}

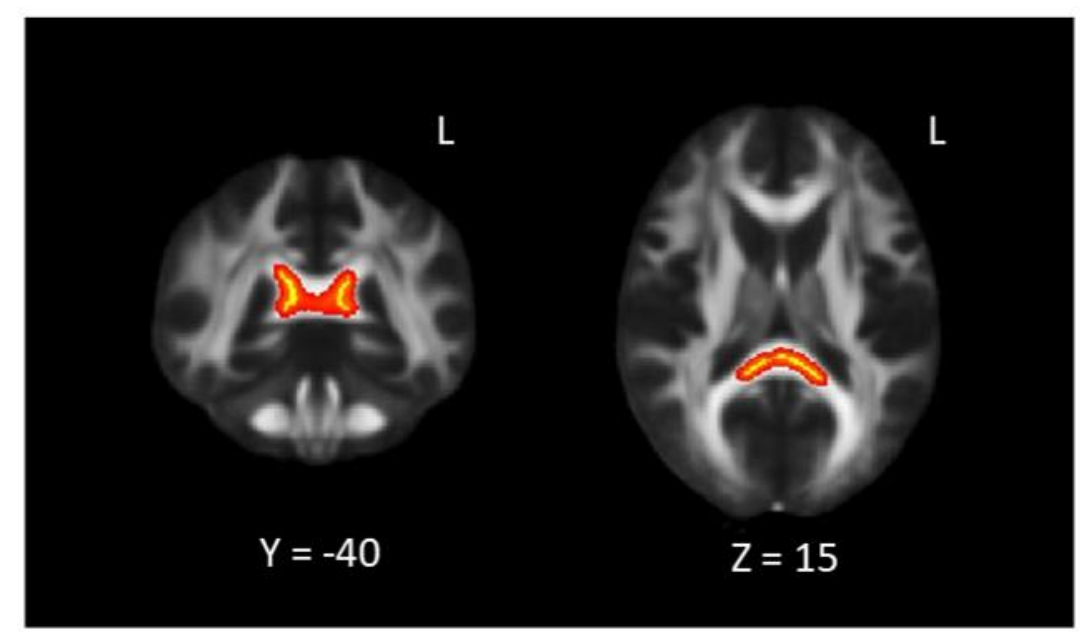

Figure 1. The TBSS image shows WM differences (FA) across the 3 groups ( $F$-test). Significant clusters are indicated in red-yellow at $p<0.05$, FWE corrected and are projected on a standardized diffusionweighted image (FMRIB58_FA). $Y$ and $Z$ indicate the coordinates of the image slices in $\mathrm{mm}$. 


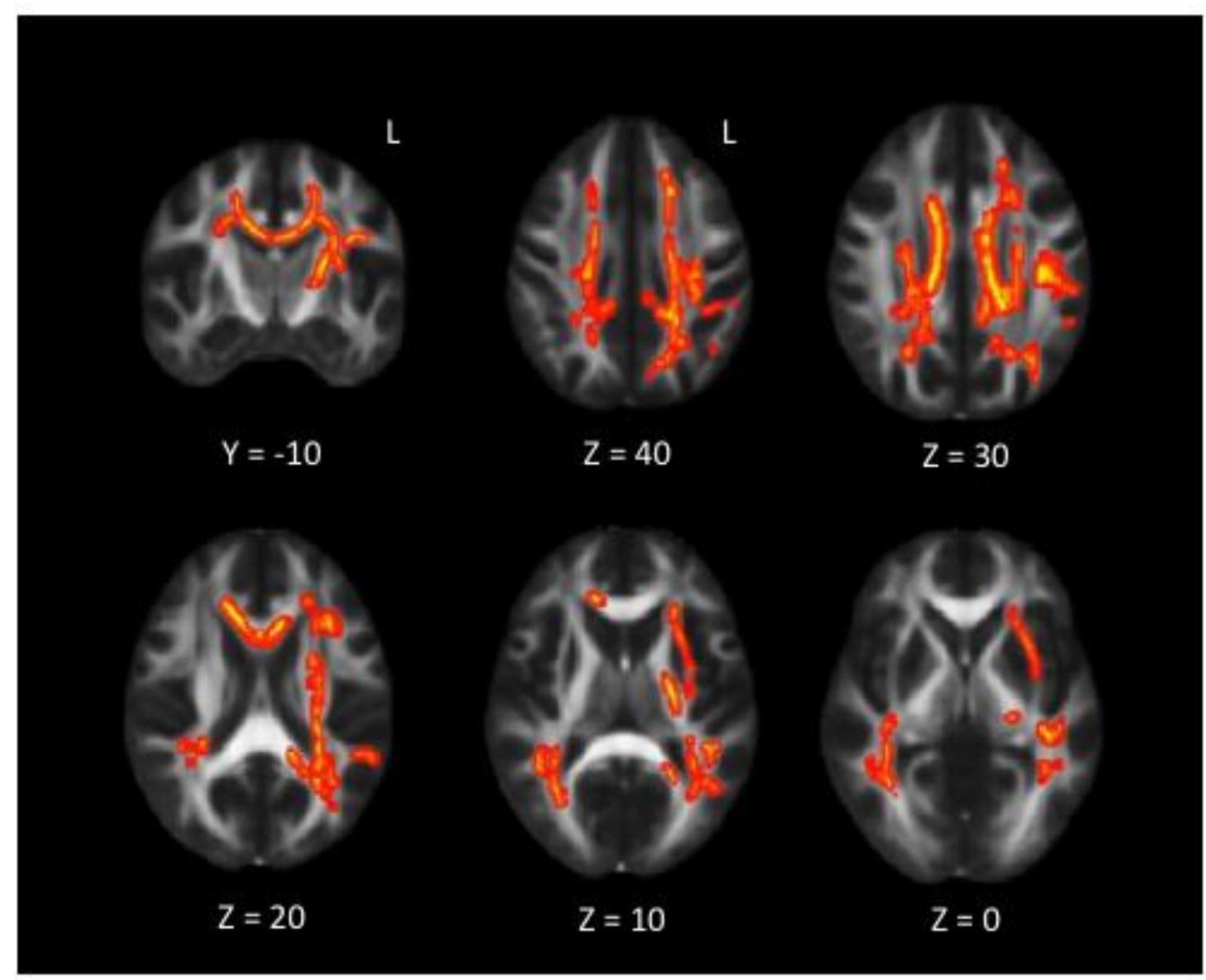

Figure 2. The TBSS image shows higher WM integrity (FA) for patients with catatonia compared to patients without catatonia. Significant clusters are indicated in red-yellow at $p<0.05$, FWE corrected and are projected on a standardized diffusion-weighted image (FMRIB58_FA). Y and Z indicate the coordinates of the image slices in $\mathrm{mm}$.

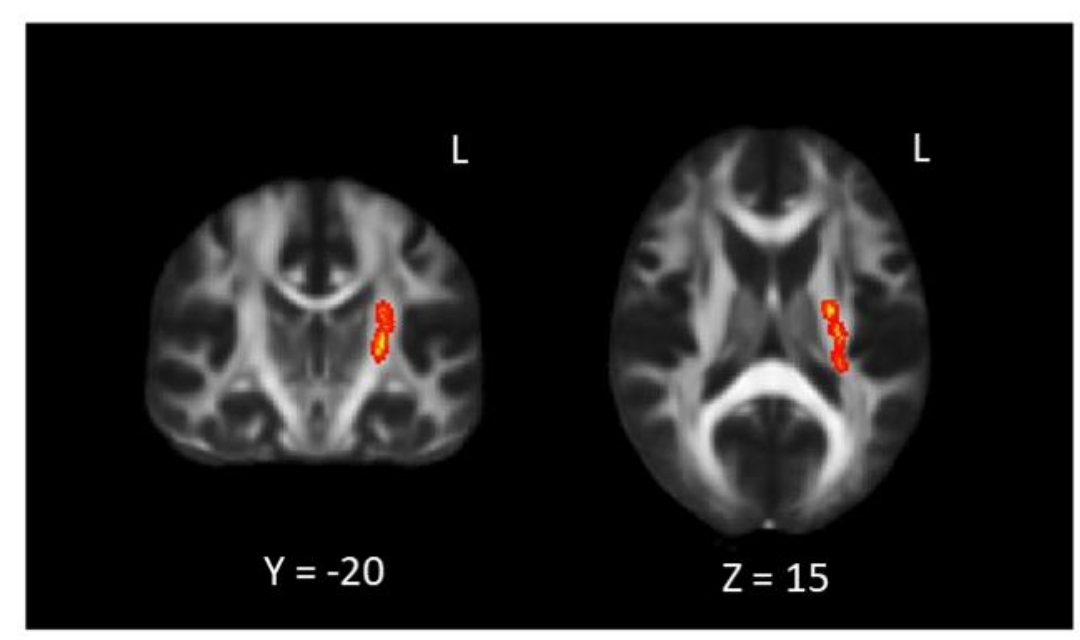

Figure 3. The TBSS image shows higher WM integrity (FA) for patients with catatonia compared to healthy controls. Significant clusters are indicated in red-yellow at $p<0.05$, FWE corrected and are projected on a standardized diffusion-weighted image (FMRIB58_FA). Y and Z indicate the coordinates of the image slices in $\mathrm{mm}$. 